\title{
La place des salariés dans l'entreprise de demain : que cache la rationalisation des entreprises françaises?
}

Nicolas Baltazar, Paris, l'Harmattan, coll. Pour Comprendre, 2012, 96 p., $12 €$

Mikaël Gléonnec

\section{OpenEdition}

\section{Journals}

Édition électronique

URL : http://journals.openedition.org/communicationorganisation/3823

DOI : 10.4000/communicationorganisation.3823

ISSN : 1775-3546

Éditeur

Presses universitaires de Bordeaux

Édition imprimée

Date de publication : 1 juin 2012

Pagination : 217-220

ISBN : 978-2-86781-827-9

ISSN : 1168-5549

Référence électronique

Mikaël Gléonnec, «La place des salariés dans l'entreprise de demain : que cache la rationalisation des entreprises françaises? », Communication et organisation [En ligne], 41 | 2012, mis en ligne le 29 mars 2013, consulté le 22 septembre 2020. URL : http://journals.openedition.org/

communicationorganisation/3823 ; DOI : https://doi.org/10.4000/communicationorganisation.3823 
personnelle ne tient pas » contredit l'auteur p. 122, faisant ainsi valoir la thèse de l'individualisme méthodologique.

Le projet de l'auteur est dans la recherche d'une synthèse. "Il nous apparaît par conséquent nécessaire de chercher un modèle d'intégration qui soit susceptible à la fois de bien prendre en compte le poids contraignant des structures et la liberté individuelle, même si celle-ci peut être également limitée par le contexte » (p. 125).

C'est ainsi que l'auteur fait appel à Anthony Giddens et à sa théorie de la structuration qui tente une synthèse où "la constitution de la société s'articule pour lui dans l'ensemble des pratiques sociales qui dépassent l'expérience individuelle et singulière de l'action sans se résumer forcément à un ensemble social, à une conception holiste de la société» (p. 127).

Dans cette réflexion sur les interactions entre les acteurs, la technologie et l'organisation, c'est la question du projet qui émerge et de la nécessité de penser le monde dans sa dynamique processuelle. "Nous ne sommes plus intéressés par une photographie figée de l'organisation, par une cartographie à l'instant $T d u$ système d'information mais par la dynamique processuelle et récursive qui s'établit entre ces trois concepts (acteur, technologie, organisation)» (p. 140).

L'auteur ouvre alors un chapitre sur les dynamiques sociales du changement organisationnel où là encore, avec beaucoup d'érudition, les auteurs et les problématiques clefs de ce concept sont exposés.

Le dernier chapitre aborde, quant à lui, la notion de projet d'innovation organisationnelle à l'aune de l'espace, du temps et de leur mutation.

Benoît Cordelier est d'une exigence théorique tout à fait exemplaire. Ethnométhodologie, pragmatisme, fonctionnalisme, individualisme méthodologique... courants de pensée, concepts et mise à distance critique occupent les pages de cet ouvrage passionnant et didactique qui présente un état des auteurs mobilisés actuellement dans le champ de la communication des organisations.

Bruno Chaudet

\section{Nicolas BALTAZAR, La place des salariés dans l'entreprise de demain : que cache la rationalisation des entreprises françaises ?, Paris, l'Harmattan, coll. Pour Comprendre, 2012, 96 p., $12 €$}

Nicolas Baltazar est titulaire du master « communication et ressources humaines » de l'Université Paris XIII, obtenu après une licence en sciences de l'information et de la communication à Paris X. Il est aujourd'hui chargé de recrutement dans une SSII. Passionné par les domaines de la communication, de la sociologie du travail et de la sociologie des organisations, il a souhaité poursuivre le travail commencé dans le cadre de son mémoire de master en réalisant un ouvrage qui présente de manière synthétique et accessible ses 
observations et ses analyses. Cette démarche l'a orienté vers la collection «Pour Comprendre » de l'Harmattan, dirigée par un comité éditorial composé de Professeurs d'Université de différents champs des sciences humaines et sociales, dont l'objectif est d'apporter au lecteur un éclairage synthétique sur une question donnée et de lui permettre d'aller plus loin grâce à une sélection bibliographique.

Le livre comprend trois chapitres qui s'enchaînent et se complètent de manière cohérente : l'un expliquant les principaux concepts sur lesquels repose l'analyse, le second présentant les différentes formes de rationalisation observables dans les entreprises, le troisième précisant la question de la rationalisation des relations informelles dans le cadre du travail collaboratif. Dès l'introduction, Nicolas Baltazar pose le paradoxe qui sera le pivot de l'ouvrage : celui d'une volonté managériale de rationalisation des pratiques informationnelles, communicationnelles et collaboratives qui relevaient, jusqu'alors, de la sphère informelle, alors que c'est dans cette même sphère que les salariés trouvent l'espace de liberté nécessaire pour que prennent corps ces relations complexes. Confronté à une injonction de plus en plus forte à formaliser, à informer, à communiquer, mais aussi à adhérer et à s'impliquer, l'individu se voit, en quelque sorte, dépossédé de la dimension sociale de son activité. Cette "instrumentalisation du social » est assortie d'un contrôle organisationnel qui, même s'il ne se dévoile pas comme tel, se révèle dans les principes de transparence, de responsabilisation, d'autorégulation et de valorisation des initiatives mis en avant dans le discours managérial. Ce sont donc des salariés en liberté surveillée qui sont incités à utiliser des outils collaboratifs qui s'inspirent directement de ceux qui ont fait le succès du Web 2.0 (réseaux sociaux, wikis, bases de connaissances partagées ), dont l'usage repose pourtant, initialement, sur la liberté d'adhérer à des communautés et de participer à leurs activités. L'introduction de ces technologies dans l'entreprise illustre parfaitement la tentative d'instrumentalisation du social à laquelle sont soumis les individus dans ce que Nicolas Baltazar appelle l'« organisation 2.0 » (p. 9). Il s'agit sans doute là de l'axe le plus novateur développé dans l'ouvrage.

L'auteur fait appel au concept de rationalisation tel que le définit Max Weber, en tant que processus de structuration et d'homogénéisation du rapport au monde au sein de la société sur la base de représentations et de logiques d'action communes. Pour Weber, l'idéologie capitaliste conduirait ce processus à instituer une " rationalité en efficacité ", selon laquelle les objectifs et les moyens mis en œuvre pour les atteindre seraient essentiellement justifiés par leur efficacité au regard de la logique économique dominante. Ainsi, le processus de rationalisation à l'œuvre dans les entreprises serait à l'origine d'une instrumentalisation des relations sociales, l'individu étant envisagé en tant que facteur de production, devant être utilisé et contrôlé dans un but d'efficacité productive. Dans ce cadre, Nicolas Baltazar perçoit les dispositifs 
de travail collaboratif et de gestion des connaissances comme des moyens mis en place pour capter, capitaliser et redistribuer ce qui peut être valorisable dans les relations humaines : les informations, les savoirs, les idées nouvelles, mais aussi les dynamiques relationnelles nécessaires à l'intelligence collective. L'individu serait alors dépossédé de ce qu'il sait, de ce qu'il pense et de ce qu'il imagine. Il serait également, en quelque sorte, dépossédé du lien social qu’il entretient dans le cadre de ses activités professionnelles, celui-ci apparaissant, lui aussi, comme un facteur de production devant être stimulé, analysé et réutilisé. Luutilisation des données ainsi captées fait ensuite l'objet de procédures auxquelles les salariés doivent se soumettre : «In fine, on veut que l'employé soit régulier dans son activité, de même niveau que ses collègues, répétitif dans ses tâches, ne devenant plus qu'un simple exécutant. Cela est donc paradoxal avec cet engouement commun de rendre l'employé autonome et force de proposition » (p.31).

Face à ce processus de rationalisation, les salariés sont capables de résistance lorsque les moyens d'information et de communication et les compétences dont ils disposent leur permettent de contourner les dispositifs de contrôle et de captage mis en place par l'entreprise (p.44). Cette résistance, même si l'auteur ne le précise pas directement, s'avère salutaire pour les organisations du fait de l'impossibilité de codifier certaines compétences clés et de « cloner » le lien social (l'impossibilité, par exemple, de dupliquer la confiance entre collaborateurs, ou les relations amicales).

L'exemple des réseaux sociaux d'entreprise (pp.50-62) illustre la tentative masquée de détournement, par les entreprises, des outils et des modes de collaboration spontanée propres au Web 2.0 à des fins de rationalisation. Il met également en évidence, de manière flagrante, les limites de cette rationalité productive : «(...) Les réseaux d'entreprise sont des outils ayant pour conséquence la création de communautés quasi artificielles. Et même si elles existent de façon informelle, elles deviennent dès à présent formalisées et visibles de tout le monde sur un support informatique dédié. » (p. 51). Les résistances à ces outils mériteraient qu'on s'y attarde davantage : comment l'individu se met-il en scène sur les réseaux sociaux d'entreprise? Qu'est-il prêt à partager réellement ? Que ne partage-t-il qu'en apparence, jouant le jeu voulu par l'entreprise sans vraiment s'investir ? Utilise-t-il les réseaux sociaux dont il fait partie à titre privé, en dehors de l'entreprise, à des fins professionnelles ?... Autant de questions qui restent en suspens dans l'ouvrage, pour lesquelles quelques éléments de réponse auraient pu être trouvés dans des travaux existants (par exemple dans l'article de Béatrice Galinon-Mélénec sur la relation entre réseaux sociaux et DRH, paru dans le n 37 de la revue Communication \& Organisation).

Pour conclure, rappelons quel était l'objectif poursuivi par Nicolas Baltazar en publiant ce livre : partager avec le plus grand nombre ses observations et ses analyses, avec ses mots, de façon à questionner le lecteur, à stimuler sa curiosité et son envie d'explorer plus en avant la thématique abordée. Sur 
ce point, l'objectif semble atteint. Cela permet de pardonner un manque de rigueur scientifique et rédactionnelle qui pourrait décontenancer certains lecteurs habitués à lire des travaux de recherche. Enfin, cet ouvrage mériterait sans doute une suite, dans le cadre d'une démarche scientifique plus structurée.

Mikaël Gléonnec 